\title{
When will small molecule lactate dehydrogenase inhibitors realize their potential in the cancer clinic?
}

\author{
"The current pace of progress in search of potential \\ drug-like small molecules pave a realistic optimism that \\ in near future...will provide new hope and safe strategy \\ for the treatment of cancer."
}

First draft submitted: 24 March 2017; Accepted for publication: 5 April 2017; Published online: 19 July 2017

Keywords: cancer metabolism • drug discovery • hLDH5 inhibitors • tumor glycolysis - Warburg effect

Reaching beyond the success in development of anticancer drugs meant to precisely focus first on one hallmark of cancer and the production of a novel type of small molecules that are selective and specific for cancer treatment. Safety is a major challenge than the efficacy in the drug development processes of small molecules, for both the academic research and pharmaceutical industries. From a metabolic assessment, unlike normal cells, the most characteristic feature of cancer cells is an unusual metabolic profile exhibiting a high rate of glucose consumption and lactate production due to increased glycolytic activity to supply them sufficient energy and metabolites. This phenomenon, known as the 'Warburg effect' described the fact that the metabolic shift toward aerobic glycolysis is largely accepted as a key metabolic hallmark of cancer cells [1] This metabolic alteration is independent of the availability of oxygen, resulting in increased levels of lactate and lower ATP production. Additionally, this metabolic trait provides a selective advantage for cancer cells to grow and proliferate and to support biomass synthesis even in the hypoxic environment. In fact, the 'glucose addiction' of cancer cells is considered to be an Achilles heel of cancer because of the highly susceptible nature of cancer cells to glucose withdrawal $[2,3]$.

\section{Is human lactate dehydrogenase-5 an innovative anticancer target?}

Recently, researchers from both academia and industries, have renewed interest in development of anticancer drugs by targeting human lactate dehydrogenase-5 (hLDH-5) and to explore the role of hLDH-5 in cancer initiation, growth and maintenance and its utility as a tumor biomarker. hLDH-5 enzyme occupies a crucial position in the Warburg effect and catalyzes the reduction of pyruvate coupled with the oxidation of nicotinamide adenine dinucleotide dehydrogenase (NADH) to form lactate and $\mathrm{NAD}^{(+)}$at the end in the final step of the glycolytic pathway. The $\mathrm{NAD}^{(+)}$thus obtained to determine the continuity of glycolysis [4]. Till date, the hLDH-5 isoform received a position in the list of highly promising hallmark of cancer, in other words, highly promising targets for cancer drug development and cancer treatment due to following main crucial facts such as: hLDH-5 isoform holds significant role as a metabolic checkpoint in the cancer glycolytic pathway; associations of this enzyme with the activation of some proto-oncogenes; its link with the maintenance of invasiveness and metastatic potential; and finally connection of hLDH-5 isoform to chemoresistance of cancer cells $[5,6]$.

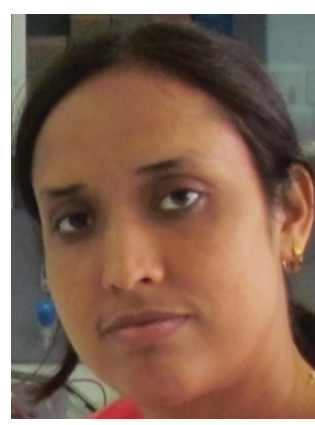

Reshma Rani

Amity Institute of Biotechnology, Amity University, Noida, UP 201313, India Author for correspondence: Tel.: +91 7536843982 reshudcy@gmail.com

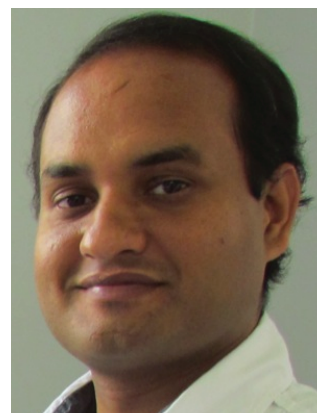

Vinit Kumar

Author for correspondence:

Amity Institute of Molecular Medicine \& Stem Cell Research, Amity University, Noida, UP 201313, India

vinitiitr@gmail.com 


\section{Is hLDH-5 a safe target?}

In our point of view, hLDH-5 isoform has significant functional roles in the development and survival of wide range of human cancers; thus, the total $\mathrm{LDH}$ activity and LDH isoform patterns could be utilized for diagnostic and prognostic significance and in neoplastic diseases. Kanno et al. found that the probable side effect might be expected due to the inhibition of hLDH-5 enzyme only in those people who have hereditary deficiency of the $l d h$ - $a$ gene leading to the lack of a subunit of hLDHA, as hLDH-5 comprises of four subunits of hLDHA. People with an $l d h-a$ gene deficiency may suffer myoglobinuaria due to muscle damage only after intense anaerobic exercise without showing any side effect under ordinary circumstances [7]. Overall, the inhibition/knockdown of hLDH-5 by shRNA, natural products or small molecules offered the definite confirmation of the crucial role of hLDH-5 in tumor initiation, development, maintenance and chemoresistance against therapy. Among the isoforms of LDH enzyme and its closely associated enzymes, the hLDH-5 enzyme has well defined and preserved structure with small structural changes, however these changes in the amino acid sequence are very useful for design and construction of small molecules able to modulate the catalytic potential [8]. Taking together, all these facts support the inhibition of hLDH-5 as a safe approach to create a new anticancer drug for clinical applications.

\section{Prospect for potential small molecule inhibitors of hLDH-5 entering into the clinic}

Small molecule drug discovery via target-based approaches are being widely employed to identify lead candidates for the development of hLDH-5 inhibitors drugs. Various small molecules including natural as well as synthetic molecules exhibiting specific inhibitory activity against hLDH-5 have recently been identified and tested in preclinical studies [9,10]. The small molecule based drug discovery approach targeting hLDH-5 was further confirmed by the fact that suppression of hLDH- 5 by small drugs could be utilized to kill cancer cells selectively, through apoptosis induction, irrespective of cancer cell p53 status and as a part of a combinatorial approach with redox-sensitive anticancer drugs via a novel p53/NAD $(\mathrm{H})$-dependent mechanism [11]. Small molecules named as NHI derivatives $N$-hydroxyindole derivatives endowed strong synergistic inhibitory effect on both enzyme as well as cell-based assays and also inhibited spheroid growth, cell migration and invasion in pancreatic cancer cells in combination with gemcitabine. These results offer a new hope in the development of new potent hLDH-5 inhibitors and their utilization in combination therapy for can- cer treatment [12]. Very recently, the role and therapeutic potential of hLDH-5 enzyme in brain tumors has been investigated and found significant involvement of hLDH-5 in brain tumors and glioblastoma multiform. This study proved that inhibition of hLDH-5 by small molecules could be an efficient strategy to target and control the glioblastoma multiform-derived cancer stem cells. hLDH-5 inhibition via small molecules has been further confirmed as a vital approach for drug design and discovery for cancer treatment and promising pharmacological target for anti-CSC (anti-cancer stem cell) therapies $[13,14]$. A large variety of small molecules showing selective hLDH5 inhibitory activity have been discovered and potent hLDH-5 inhibitors including GNE-140, FX11, galloflavin and RNAi have been studied preclinically as anticancer agents. Besides, several others hLDH-5 inhibitors such as gossypol (natural product), galloflavin, $N$-hydroxyindole derivatives, chimeric molecules and quinolone sulfonamide inhibitors have been developed [9]. However, none of these compounds were approved for clinical uses. Development of hLDH-5 inhibitors as anticancer drugs that can be used in clinics is not easy, nevertheless it is not impossible. However, the development of drugs will depend on the advancement in various research and training programs and multidisciplinary aspects of bridging chemistry, chemical biology, chemoinformatics and bioinformatics. With these advances in hand, including with close linking of genetics and chemistry, create a new hope for the generation of new hLDH-5 inhibitors as drug-like candidates.

Although, this editorial is not intended to give a definitive answer to the entitled question, "When will small molecule LDH inhibitors realize their potential in the cancer clinic?". We offer a platform for further valuable discussion and motivation to fill the gap of unexplored small drug-like molecules and potential strategies to fight cancer. Concisely, cancer is not a single disease, but a collection of many diseases and disorders encompassing several complex processes. Thus, treatment of cancer requires strong discussion, wide range of associated research that cannot be limited to single protocol. Computer-aided drug design, synthetic chemistry and chemical biology, all together could be valuable approach to discover a potent drug-like molecule that counteract energetics of cancer cell by targeting hLDH-5 enzyme and entered into clinical trial. Targeting tumor glycolysis together with mitochondrial metabolism with drug combinations holds immense promises. The current pace of progress in search of potential drug-like small molecules pave a realistic optimism that in near future, the introduction of potential hLDH-5 inhibitors into clinical practice will provide new hope and safe strategy for the treatment of cancer. 


\section{Acknowledgements}

Authors are thankful to Amity University, Noida, India. Authors are also thankful to DST-SERB, government of India.

\section{Financial \& competing interests disclosure}

The authors have no relevant affiliations or financial involvement with any organization or entity with a financial

\section{References}

1 Warburg O. On the origin of cancer cells. Science 123, 309-314 (1956).

2 Cantor JR, Sabatini DM. Cancer cell metabolism: one hallmark: many faces. Cancer Discov. 2, 881-898 (2012).

3 Seyfried TN. Energetics of normal cells and cancer cells. In: Cancer as a Metabolic Disease on the Origin: Management and Prevention of Cancer. Wiley \& Sons, NJ, USA (2012).

4 Martinez-Outschoorn UE, Peiris-Pagés M, Pestell RG, Sotgia F, Lisanti MP. Cancer metabolism: a therapeutic perspective. Nat. Rev. Clin. Oncol. 14, 11-31 (2017).

5 Stefano GD, Manerba M, Ianni LD, Fiume L. Lactate dehydrogenase inhibition: exploring possible applications beyond cancer treatment. Future Med. Chem. 8, 713-725 (2016).

6 Li XM, Xiao WH, Zhao HX. Discovery of potent human lactate dehydrogenase A (LDHA) inhibitors with antiproliferative activity against lung cancer cells: virtual screening and biological evaluation. Med. Chem. Commun. 8, 599-605 (2017).

7 Kanno T, Sudo K, Maekawa M, Nishimura Y, Ukita M, Fukutake K. Lactate dehydrogenase M-subunit deficiency: a new type of hereditary exertional myopathy. Clin. Chim. Acta 173, 89-98 (1988).

8 Lagana G, Barreca D, Calderaro A, Bellocco E. Lactate dehydrogenase inhibition: biochemical relevance interest in or financial conflict with the subject matter or materials discussed in the manuscript. This includes employment, consultancies, honoraria, stock ownership or options, expert testimony, grants or patents received or pending, or royalties.

No writing assistance was utilized in the production of this manuscript.

and therapeutical potential. Curr. Med. Chem. doi:10.2174/0 929867324666170209103444 (2017) (Epub ahead of print).

9 Rani R, Kumar V. Recent update on human lactate dehydrogenase enzyme 5 ( $h \mathrm{LDH} 5$ ) inhibitors: a promising approach for cancer chemotherapy. J. Med. Chem. 59, 487-496 (2016).

10 Granchi C, Paterni I, Rani R, Minutolo F. Small-molecule inhibitors of human lactate dehydrogenase 5 (hLDH5). Fut. Med. Chem. 5, 1967-1991 (2013).

11 Allison SJ, Knigh JRP, Granchi C et al. Identification of $\mathrm{LDH}-\mathrm{A}$ as a therapeutic target for cancer cell killing via (i) p53/NAD $(\mathrm{H})$-dependent and (ii) p53-independent pathways. Oncogenesis 3, e102 (2014).

12 Maftouh M, Avan A, Sciarrillo R et al. Synergistic interaction of novel lactate dehydrogenase inhibitors with gemcitabine against pancreatic cancer cells in hypoxia. Br. J. Cancer 110 , 172-182 (2014).

13 Valvona CJ, Fillmore HL, Nunn PB, Pilking GJ. The regulation and function of lactate dehydrogenase $A$, therapeutic potential in brain tumor. Brain Pathol. 26(1), 3-17 (2016).

14 Daniele S, Giacomelli C, Zappelli E et al. Lactate dehydrogenase-A inhibition induces human glioblastoma multiforme stem cell differentiation and death. Sci. Rep. 5 , 15556 (2015). 